\title{
Corrigendum: Association of heated tobacco product use and secondhand smoke exposure with suicidal ideation, suicide plans, and suicide attempts among Korean adolescents: A 2019 national survey
}

\author{
Soyoon Park', Kang-Sook Lee ${ }^{1,2}$
}

Corrigendum on:

Association of heated tobacco product use and secondhand smoke exposure with suicidal ideation, suicide plans, and suicide attempts among Korean adolescents: A 2019 national survey

By Soyoon Park, Kang-Sook Lee

Tobacco Induced Diseases, Volume 19, Issue September, Pages 1-11, Publish date: 21 September 2021

DOI: https://doi.org/10.18332/tid/140824

The manuscript type is 'Research Paper' instead of 'Review Paper'.

AFFILIATION

1 Department of Public Health, Graduate School, The Catholic University of Korea, Seoul, Korea

2 Department of Preventive Medicine, College of Medicine, The Catholic University of Korea, Seoul, Korea

CORRESPONDENCE TO Kang-Sook Lee. Department of Preventive Medicine, College of Medicine, The Catholic University of Korea, 222 Banpo-daero, Seocho-gu, Seoul 06591, Korea. E-mail: leekangs@catholic.ac.kr

\section{KEYWORDS}

heated tobacco product, SHS, smoking, suicide, adolescents

Received: 16 November 2021 Accepted: 19 November 2021 